\title{
Co-administration of erythropoietin and iron complex improves late-phase liver regeneration
}

\author{
Ji-Yoon Kim ${ }^{1}$, Dongho Choi ${ }^{2}$, Joohwan Kim ${ }^{3}$, Young-Myeong Kim ${ }^{3}$, Hyunyoung Lim ${ }^{1}$, Jeong Min Sung ${ }^{1}$, Min Kyu Lee ${ }^{1}$, \\ Yoo Jin Choung ${ }^{1}$, Ji Hee Chang ${ }^{1}$ \& Mi Ae Jeong ${ }^{1, *}$ \\ ${ }^{1}$ Department of Anesthesiology and Pain Medicine, Hanyang University Hospital, Seoul 04763, ${ }^{2}$ Department of Surgery, Hanyang \\ University Hospital, Seoul 04763, ${ }^{3}$ Department of Molecular and Cellular Biochemistry, Kangwon National University, School of Medicine, \\ Chuncheon 24341, Korea
}

\begin{abstract}
Erythropoietin and iron have individually shown beneficial effects on early-phase liver regeneration following partial hepatectomy (PHx); however, there are limited data on the combined effect on late-phase liver regeneration after PHx. Here we examined combined effects of recombinant human erythropoietin (rhEPO, 3,000 IU/kg) and iron isomaltoside (IIM, $40 \mathrm{mg} / \mathrm{kg}$ ) on late-phase liver regeneration following PHx and investigated the possible underlying mechanism. Rats administrated with rhEPO showed significantly higher liver mass restoration, interleukin-6 (IL-6, a hepatocyte mitogen) levels, and Ki-67-positive hepatocytes on day 7 after PHx than saline-treated controls. These beneficial effects were further enhanced on days 7 and 14 by co-treatment with IIM. This combination also significantly improved liver function indices, such as increased albumin production and decreased bilirubin levels, but did not alter serum levels of toxic parameters, such as aspartate transaminase and alanine transaminase. This study demonstrates that the combination of rhEPO and IIM synergistically improves late-phase liver regeneration and function after PHx, probably by promoting IL-6-mediated hepatocyte proliferation without adverse effects. Thus, this combination treatment can be a potential therapeutic strategy for patients undergoing resection for hepatic malignancies. [BMB Reports 2020; 53(3): 148-153]
\end{abstract}

\section{INTRODUCTION}

Liver regeneration is a coordinated and homeostatic process that rapidly recoups lost functional liver mass, allowing performance of extensive resection as a curative option for

${ }^{*}$ Corresponding author. Tel: +82-2-2290-8695; Fax: +82-2-22998692; E-mail: macheong@hanyang.ac.kr

https://doi.org/10.5483/BMBRep.2020.53.3.160

Received 12 June 2019, Revised 6 July 2019, Accepted 23 July 2019

Keywords: Erythropoietin, Hepatic function, Interlukin-6, Iron isomaltoside, Liver regeneration patients with primary or secondary hepatic cancers. However, as excessive resection can increase risk of post-operative liver failure $(1,2)$ reducing or minimizing further damage to the remnant liver is essential. Additionally, it is also important to successfully stimulate regenerative function and capability of remaining liver tissue after hepatectomy by exogenously supplying or endogenously inducing mitogenic and growth factors (3-7).

There are two types of cells responsible for liver regeneration: liver stem cells and hepatocytes. Liver stem cells participate in compensatory regeneration of the liver damaged by viral and chemical insults, and hepatocytes are essential for liver growth or restoration after resection. Because hepatocytes, as fully differentiated and quiescent cells, exist in the $\mathrm{G}_{0}$ phase of the cell cycle (8), liver regeneration after resection requires a priming event that would allow hepatocytes to re-enter the cell cycle from the $\mathrm{G}_{0}$ to the $\mathrm{G}_{1}$ phase, subsequently leading to hepatocyte proliferation. Interleukin-6 (IL-6), tumor necrosis factor- $\alpha$ (TNF- $\alpha$ ), and hepatocyte growth factor (HGF) are potential priming cytokines (9); however, the liver regeneration process can be triggered by multiple signaling cascades activated by coordinated actions with other cytokines, growth factors, and matrix remodeling, and are regulated by several positive and negative cross-talks among growth-related signals $(8,9)$.

The hematopoietic growth factor erythropoietin (EPO) not only stimulates erythropoiesis in bone marrow (10) but also has other multiple functions, including anti-apoptotic and hepatoprotective actions (11). Additionally, administration of recombinant human EPO (rhEPO) stimulates hepatic proliferation and liver regeneration after partial hepatectomy (PHx) (3-5). Similarly, iron supplementation also increases hepatocyte proliferation after $\mathrm{PHx}(6)$, probably by enhancing catalytic activity of ribonucleotide reductase (12). Although rhEPO and iron individually improve early-phase liver regeneration in hepatectomized rats, the combination effect and underlying mechanism have not been clearly investigated in late-phase liver regeneration after $\mathrm{PHx}$.

The purpose of this study was to assess combined effects of rhEPO and iron isomaltoside (IIM, an iron-carbohydrate

ISSN: 1976-670X (electronic edition)

Copyright (C) 2020 by the The Korean Society for Biochemistry and Molecular Biology

c) This is an open-access article distributed under the terms of the Creative Commons Attribution Non-Commercial License (http://creativecommons.org/licenses/by-nc/4.0) which permits unrestricted non-commercial use, distribution, and reproduction in any medium, provided the original work is properly cited. 
complex) on late-phase liver regeneration, function, and toxicity in a rat model of $70 \% \mathrm{PHx}$, because, to our knowledge, this has never been done. Our results will assist in clinical development of a therapeutic strategy for hepatic failure resulting from a small remnant liver volume.

\section{RESULTS}

\section{Co-administration of rhEPO and IIM promotes late-phase liver restoration}

Control remnant livers reached approximately $72 \%$ of the relative liver weight on day 7 after $\mathrm{PHx}$, and this recovery effect further increased by treatment with only rhEPO and strongly augmented by co-administration of rhEPO and IIM (Fig. 1). On day 14 after $\mathrm{PHx}$, the regeneration rate of control livers reached approximately $81 \%$, and this rate was slightly increased, but not significantly, by treatment with only rhEPO and significantly enhanced by Co-administration of rhEPO and IIM (Fig. 1). Their combination also slightly increased hemoglobin and hematocrit levels by approximately $110 \%$ $(11.94 \pm 0.07$ vs. $12.98 \pm 0.35 \mathrm{~g} / \mathrm{dl}$ and $38.83 \pm 0.48$ vs. $42.38 \pm 1.07 \%$ ) only on day 14 compared with untreated control, but did not alter the numbers of white blood cells and platelets. Collectively, these results show that rhEPO promotes late-phase liver restoration after $\mathrm{PHx}$ and that this effect is further augmented by co-treatment with IIM.

\section{Combination of rhEPO and IIM prolongs late-phase hepatocyte proliferation}

To compare the relationship between liver regeneration and hepatocyte proliferation in rats co-administrated with rhEPO and IIM following PHx, we performed immunohistochemical examination of the expression of proliferation marker Ki-67 in

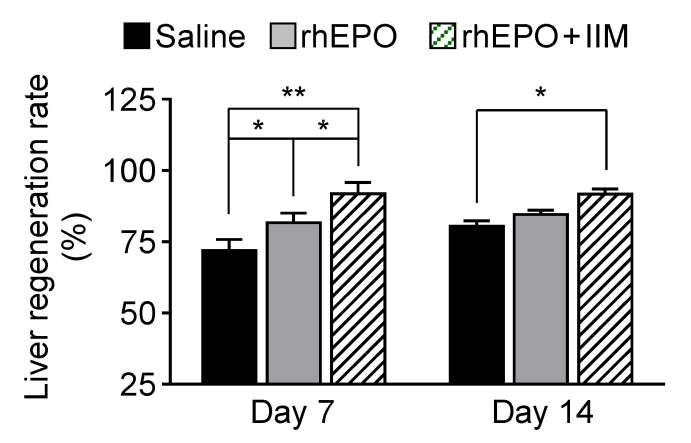

Fig. 1. Co-administration of rhEPO and IIM synergistically promotes late-phase restoration of liver mass in hepatectomized rats. Rats were subjected to $\mathrm{PHx}$ and were administrated with saline, s.c. rhEPO $(3,000 \mathrm{IU} / \mathrm{kg})$, or s.c. rhEPO in combination with i.v. IIM (40 mg/kg). Livers were removed on days 7 and 14 after liver resection. Liver regeneration rate was calculated using protocol described in Materials and Methods. $\mathrm{n}=8,{ }^{*} \mathrm{P}<0.05$ and $* * \mathrm{P}$ $<0.01$. liver tissues. On day 7 after PHx, only a few Ki-67-positive hepatocytes appeared in liver tissues from control hepatectomized rats, whereas the Ki-67-positive index significantly increased by rhEPO compared with the control, and this effect was further augmented by co-administration of rhEPO and IIM (Fig. 2A and 2B). However, Ki-67-positive cells were barely or insignificantly detected in all experimental groups on day 14 after PHx (Fig. 2A and 2B). These results suggest that co-administration of rhEPO and IIM potentially stimulates and prolongs hepatocyte proliferation, contributing to improvement of late-phase liver regeneration after $\mathrm{PHx}$.

\section{Co-treatment with rhEPO and IIM maintains high levels of IL-6 but not HGF}

Among many cytokines and growth factors, IL- 6 and HGF are major priming factors of hepatocyte proliferation $(13,14)$. We determined serum levels of IL-6 and HGF in hepatectomized rats. Serum HGF levels were not significantly altered by administration of only rhEPO or by the combination of rhEPO and IIM on days 7 and 14 after PHx compared with those in sham and saline controls (Fig. 3A). However, serum IL-6 levels increased, but not statistically significant, by rhEPO in hepatectomized rats on day 7 after surgery, and further signifi-

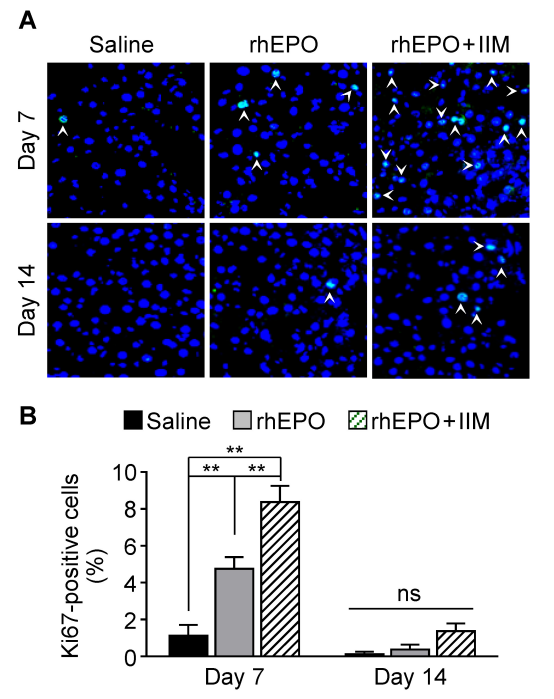

Fig. 2. Co-administration of rhEPO and IIM augments hepatocyte proliferation during the late phase of liver regeneration after $\mathrm{PHx}$. Rats were administrated with saline, rhEPO, or rhEPO in combination with IIM following PHx. Livers were removed on days 7 and 14 after liver resection. Liver tissues obtained on days 7 and 14 after PHx were immunohistochemically stained with anti-Ki-67 antibody and secondary antibody labeled with Alexa Fluor 488. Nuclei were also counterstained with DAPI. (A) Fluorescence images were captured using a confocal microscope. Arrows indicate cells stained by antibody for Ki-67 ( $\mathrm{n}=8$ ). (B) Ki-67-positive cells were quantified by counting the total number of nuclei per high-power field $(\mathrm{n}=8)$. ${ }^{* * P}<0.01$. $\mathrm{ns}$, not significant. 
A $\square$ Sham $\square$ Saline $\square$ rhEPO $\square$ rhEPO+IIM

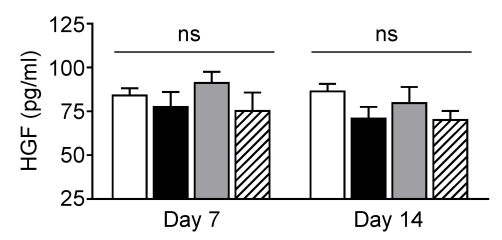

B

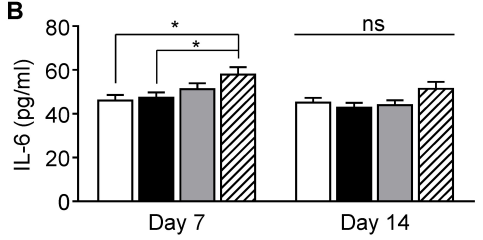

Fig. 3. Co-administration of rhEPO and IIM increases the serum levels of IL- 6 during the late phase of liver regeneration. Rats were subjected to sham operation or $\mathrm{PHx}$ and were administrated with saline, rhEPO, or rhEPO in combination with IIM. Blood samples were collected on days 7 and 14 after surgery. Serum levels of HGF (A) and IL-6 (B) were determined using ELISA kits $(\mathrm{n}=8) .{ }^{*} \mathrm{P}<0.05$. ns, not significant.

cantly enhanced by co-treatment with rhEPO and IIM; however, these effects disappeared on day 14 (Fig. 3B). The present data suggest that co-administration of rhEPO and IIM maintains high serum IL-6 levels during the late phase of liver regeneration.

\section{Co-treatment with rhEPO and IIM improves late-phase liver function}

Activities of hepatic injury the marker enzymes aspartate transaminase (AST) and alanine aminotransferase (ALT) slightly increased, but not significantly (relative to statistics), in the sera of hepatectomized rats on days 7 and 14, compared with those in sham animals, and the enzyme levels were not altered by administration with rhEPO or by the combination of rhEPO and IIM (Fig. 4A and 4B). We next examined serum levels of albumin and total bilirubin, as markers of liver function. On day 7 after $\mathrm{PHx}$, serum levels of albumin decreased in hepatectomized control rats compared with those in sham animals, and this decrease was partially recovered, but not significantly, by treatment with only rhEPO and restored to sham control levels by the co-administration of rhEPO and IIM (Fig. 4C). Conversely, serum levels of total bilirubin were significantly elevated in hepatectomized control animals on day 7 , and this increase was slightly reduced by the administration of rhEPO and further decreased to sham control levels by co-treatment with rhEPO and IIM (Fig. 4D). However, no significant differences in serum levels of albumin and total bilirubin among all groups were observed on day 14 after $\mathrm{PHx}$ (Fig. 4C and 4D). These results suggest that co-administration of rhEPO and IIM effectively restores late-phase liver function in hepatectomized rats.
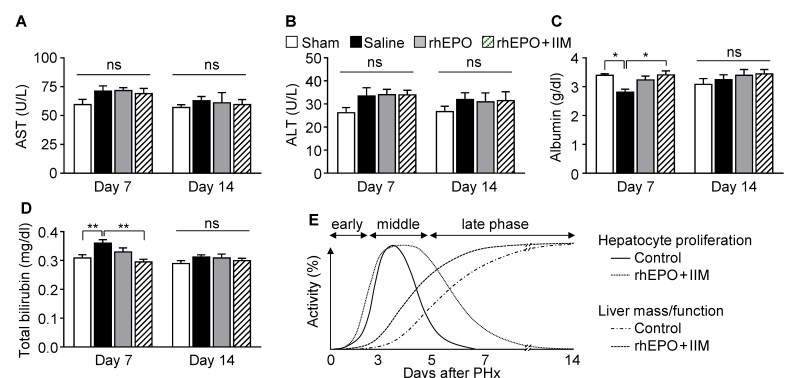

Fig. 4. Combined effect of rhEPO and IIM on the serum levels of AST, ALT, albumin, and total bilirubin during the late phase of liver regeneration. Rats were subjected to sham operation or $\mathrm{PHx}$. Hepatectomized rats were administrated with saline, rhEPO, or rhEPO in combination with IIM. Blood samples were obtained on days 7 and 14 after resection, and sera were prepared by centrifugation. Serum levels of AST (A), ALT (B), albumin (C), and total bilirubin (D) were determined using colorimetric assay kits and ELISA kits $(n=8)$. (E) Time kinetics of hepatocyte proliferation and liver regeneration in the early (hepatocyte priming), middle (hepatocyte proliferation), and late phases (liver function restoration) after co-administration of rhEPO and IIM. ${ }^{* P}<0.05$ and $* * P<0.01$. ns, not significant.

\section{DISCUSSION}

Based on the understanding of mechanisms of liver regeneration, several successful strategies have been developed to counteract post-operative liver insufficiency or post-hepatectomy liver failure (15-17). Despite successful hepatectomy, insufficient remnant liver volume and function are central factors in the etiology of fulminant liver failure after major liver resection (18). Thus, some studies have proposed potential therapeutic treatments, such as high-dose insulin therapy and portal vein embolization, which effectively increase regenerative capacity of remaining liver after resection, subsequently improving the outcome of patients undergoing liver surgery $(16,17)$.

Among many possible strategies, a number of growth factors, cytokines, and drugs have been considered as potential candidates for enhancing proliferative capability of remnant parenchymal hepatocytes (3-7). There is some evidence showing that supplementation with either rhEPO or iron improves early-phase liver regeneration and function in hepatectomized rats $(3-6,19)$. However, the combined effect of rhEPO and iron on liver regeneration has not been studied, particularly in the late phase of liver regeneration after PHx. Similar to previous studies in the early phase of liver regeneration (3-5), our study shows the beneficial effect of rhEPO on hepatocyte proliferation and liver mass restoration in the late phase of liver regeneration following $\mathrm{PHx}$. More notably, the effect of rhEPO was further improved by co-administration with IIM, suggesting a synergistic action between rhEPO and IIM in late-phase liver regeneration.

Because hepatocytes are quiescent, highly differentiated cells, they are not normally proliferative (8). Special stimulants 
are required for priming hepatocytes to re-enter the cell cycle into the $\mathrm{G}_{1}$ phase from the $\mathrm{G}_{0}$ phase, and to initiate cell proliferation (9). Priming and auxiliary factors activate hepatocytes in the remnant liver to promote the proper progression of the cell cycle by triggering the signaling pathways of cell proliferation. These pathways are stimulated by several cytokines and growth factors, including IL-6, TNF- $\alpha$, and HGF, which are endogenously produced after hepatectomy $(13,14)$. Thus, inadequate production and maintenance of those factors delay liver regeneration and can cause hepatic failure. This unfavorable phenomenon can be overcome by means of exogenous supplementation of hepatocyte-activating factors such as rhEPO and IL-6 $(3,13)$. Activity of rhEPO in liver regeneration was reported to be synergistically or additively elevated by co-treatment with the antioxidant curcumin (7), suggesting that there are several modes of action, e.g., positive or synergistic cooperation, among the growth-related signaling pathways. Our data shows that the combination of rhEPO and IIM maintains high levels of circulating IL- 6 in the late phase of liver regeneration, which plays a pivotal role in priming hepatocytes for proliferation in the early and late phases of liver regeneration $(8,20)$. This suggests that the combination of rhEPO and IIM potentiates late-phase liver regeneration and function through a mechanism of hepatocyte proliferation associated with long-term maintenance of high IL-6 levels after PHx.

Hepatocytes specifically synthesize albumin, urea, and $\alpha$-fetoprotein, as well as take up bilirubin from blood circulation, which subsequently conjugates with glucuronic acid and is finally secreted into bile (21). Indeed, serum albumin levels decreased by an impaired liver function in hepatectomized mice (22), whereas serum bilirubin levels increased by insufficient liver mass and function (23). Thus, changes in serum levels of albumin and total bilirubin are considered potential indices of the progression of liver regeneration after $\mathrm{PHx}$. Our data shows that rhEPO resulted in an increase in serum albumin levels and a decrease in serum total bilirubin levels in the late stage of liver regeneration, and their circulating levels were further restored to levels of the sham group by the co-administration of IIM. These results suggest that the combination of rhEPO and IIM promotes hepatic maturation and function during the late phase of liver regeneration (Fig. 4D).

As the hepatocytic amino acid-metabolizing enzymes AST and ALT are secreted under conditions of liver injury or damage, circulating activities of both enzymes have been used as indicators of liver injury and toxicity. In this study, we found that rhEPO alone or in combination with IIM did not significantly increase serum levels of AST and ALT in the late phase of liver regeneration after $\mathrm{PHx}$, suggesting that the combination treatment does not induce hepatotoxicity. So, we conclude that co-administration of rhEPO and IIM stimulates hepatocyte proliferation and maturation during the late phase of liver regeneration without causing hepatotoxicity.
A number of studies have shown that rhEPO treatment is safe and well tolerated in patients with anemia and stroke, improving clinical remission of anemia and neurologic symptoms $(24,25)$. Although iron is an essential component or a cofactor of many critical proteins and enzymes, such as hemoglobin, cytochrome P450s, ribonucleotide reductase, and many other enzymes, biological free iron causes toxicity by generating reactive oxygen species or free radicals through redox-based reactions. Despite the presence of a biological detoxification mechanism, intravenous infusion or administration of free iron is prohibited. Thus, only a few types of iron complex can be intravenously administrated in the form of iron-carbohydrate complex containing a polynuclear $\mathrm{Fe}^{3+}$ hydroxide that is stabilized by carbohydrate ligands (26), because it is chemically inert and does not generate reactive oxygen species. In this study, rats were intravenously administrated with IIM (Monofer ${ }^{\mathbb{R}}$ ), which had been recently developed for treating anemia across different therapeutic strategies with a pharmacological safety profile $(27,28)$. We also found that IIM combined with rhEPO did not cause hepatotoxicity in the late phase of liver regeneration.

In summary, our results show that rhEPO administration enhances liver regeneration and function to some extent through maintenance of high levels of serum IL-6 and subsequent stimulation of hepatocyte proliferation for a prolonged period until the late phase of liver regeneration. Notably, these effects were further significantly augmented by co-treatment with IIM, without inducing hepatotoxicity. This strategy may offer potential benefits for the enhancement of liver regeneration and recovery in patients with hepatic malignancy after $\mathrm{PHx}$ and in donor patients undergoing living-donor liver transplantation.

\section{MATERIALS AND METHODS}

\section{Animals}

Inbred male Sprague-Dawley rats weighting 300-350 g (7-8 weeks old, Orient Bio Inc., Sungnam, South Korea) were used in this study. The animals were housed in a specific pathogenfree animal facility with constant temperature of $21 \pm 2^{\circ} \mathrm{C}$, relative humidity of $45 \pm 15 \%$, and a 12 h-light/dark cycle and given standard food and water ad libitum. Experimental protocol was approved by the Animal Ethics Committee of Hanyang University (approval number 2017-0260A). All experimental procedures were conducted in accordance with international guidelines for care and use of laboratory animals.

\section{Experimental design}

Rats were randomly divided into a sham group and three experimental groups by using the sealed envelope method. The sham group consisted of eight rats, whereas experimental groups consisted of 16 animals per group. Each group was divided into two subgroups (8 animals in each subgroup). Experimental groups underwent a $70 \% \mathrm{PHx}$ and were treated 
with saline, subcutaneous (s.c.) rhEPO (3,000 IU/kg, Eporon ${ }^{\circledR}$; Dong-A ST Co., Ltd., Seoul, Korea), or s.c. rhEPO in combination with intravenous (i.v.) IIM (40 mg/kg, MonoFer ${ }^{R}$; Pharmacosmos ASS, Holbaek, Denmark). Rats were sacrificed on days 7 and 14 after surgery.

\section{Surgical procedure}

Rats were anesthetized through intraperitoneal administration of $80 \mathrm{mg} / \mathrm{kg}$ ketamine and $10 \mathrm{mg} / \mathrm{kg}$ xylazine, and their weight was recorded. After placing rats in the supine position, 24-G angiocatheters (BD Angiocath Plus; Becton Dickinson Medical, Singapore) were inserted into the left femoral artery for blood collection, and blood samples were used for serological and biochemical analyses. A 70\% $\mathrm{PHx}$ resecting the left and median lobes of the liver was performed according to Higgins and Anderson's method (29). Hepatectomized specimens were weighed and kept at $-80^{\circ} \mathrm{C}$. Some specimens were preserved in $3.7 \%$ formaldehyde (10\% formalin) solution. The abdomen was closed with continuous stitches using 4-0 silk sutures and cleaned with povidone iodine. Rats were given s.c. and i.v. injections of saline for the saline control group, and s.c. rhEPO $(3,000 \mathrm{IU} / \mathrm{kg})$ alone or in combination with i.v. IIM (40 mg/kg) for the treatment groups. After finishing surgical procedures, all animals were subcutaneously injected with $5 \mathrm{mg} / \mathrm{kg}$ caprofen (Ramadyl ${ }^{\mathbb{R}}$; Pfizer Inc., New York, NY, USA) to relieve pain. Rats were sacrificed on post-operative days 7 and 14, intracardiac blood was obtained for biochemical analysis. Additionally, the livers were completely excised, weighed, and divided into two pieces: one piece was kept at $-80^{\circ} \mathrm{C}$ and another in $3.7 \%$ formaldehyde solution until use.

\section{Regeneration rate}

Relative liver regeneration rate was calculated as previously described (30). Basically, body weight of rats was measured before $\mathrm{PHx}\left(\mathrm{BW}_{\text {pre-PHx }}\right)$ and at euthanasia $\left(\mathrm{BW}_{\text {euthanasia }}\right)$, and pre-operative estimation of liver weight $\left(\mathrm{ELW}_{\text {pre-PHx }}\right)$ was calculated from the relative ratio between resected liver weight and relative extent of $\mathrm{PHx}\left[\left(\mathrm{LW}_{\text {resection }} / 70\right) \times 100\right]$. Regenerated liver weight $\left(\mathrm{LW}_{\text {euthanasia }}\right)$ was measured at euthanasia. Liver regeneration rate was defined as follows: rate $(\%)=$ $\left[\left(\mathrm{LW}_{\text {euthanasia }} / \mathrm{BW}_{\text {euthanasia }}\right) /\left(\mathrm{ELW}_{\text {pre-PHx }} / \mathrm{BW}_{\text {pre-PHx }}\right)\right] \times 100$.

\section{Immunohistochemistry}

Hepatocyte proliferation was determined by means of immunohistochemical counting of Ki-67-positive cells. Liver tissues fixed in $3.7 \%$ formaldehyde solution were embedded in Optimal Cutting Tissue compound (Leica Biosystems, Richmond, IL, USA) and subsequently frozen in liquid nitrogen. Tissues were sectioned at a thickness of $10 \mu \mathrm{m}$ and washed in phosphate-buffered saline (PBS) containing $0.1 \%$ Triton X-100, followed by incubation with blocking solution (Dako North America, Carpinteria, CA, USA) for 30 minutes. The sections were incubated with primary mouse monoclonal antibody against Ki-67 (Abcam, Burlingame, CA, USA; 1:200) for two hours. After washing with PBS, the sections were incubated with Alexa Fluor ${ }^{R}$ 488-labeled goat anti-mouse immunoglobulin G (Invitrogen, Carlsbad, CA, USA) for $1 \mathrm{~h}$. Nuclei were counterstained with 4',6-diamidino-2-phenylindole (DAPI). After mounting with Mounting Medium (Dako North America), images were taken and analyzed using a confocal fluorescence microscope. The percentages of Ki-67-positive cells were quantified using Image software $(\mathrm{NIH}$, Bethesda, MD, USA).

\section{Biochemical analysis}

Blood samples were collected in tubes containing EDTA and centrifuged at 3,000 rpm at $4^{\circ} \mathrm{C}$ for $15 \mathrm{~min}$ (ScanSpeed 1730R; Labogene, Lillerød, Denmark), and sera were stored at $-80^{\circ} \mathrm{C}$ until use. Serum levels of AST and ALT were determined using a colorimetric assay kit (Asan Pharmaceutical, Seoul, Korea) according to the manufacturer's instructions. Serum levels of HGF and IL-6 were analyzed using mouse/rat HGF Quantikine enzyme-linked immunosorbent assay (ELISA) kit (cat. no. MHG00) and a rat IL-6 Quantikine ELISA kit (cat. no. R6000B), purchased from R\&D Systems (Minneapolis, MN, USA). Serum levels of albumin and total bilirubin were determined using ELISA kits purchased from Shibayagi Corporation (Gunma, Japan; cat. no. AKRAL-120) and Sigma-Aldrich (St. Louis, MO, USA; cat. no. MAK126), respectively.

\section{Statistical analysis}

Quantitative data are expressed as mean \pm standard error of samples from 8 rats in each group. Data were analyzed using GraphPad Prism 6 software (GraphPad Inc., La Jolla, CA, USA). Data met assumptions of the test, and variance between statistically compared groups was similar. Statistical differences were tested using two-way analysis of variance with HolmSidak post-hoc test. $\mathrm{P}<0.05$ was significant.

\section{ACKNOWLEDGEMENTS}

This paper was supported by the National Research Foundation of Korea (NRF) grant funded by the Korea government (MSIT) (NRF-2017R1A2B3004565).

\section{CONFLICTS OF INTEREST}

The authors have no conflicting interests.

\section{REFERENCES}

1. Belghiti J, Hiramatsu K, Benoist S, Massault P, Sauvanet A and Farges $O$ (2000) Seven hundred forty-seven hepatectomies in the 1990s: an update to evaluate the actual risk of liver resection. J Am Coll Surg 191, 38-46

2. Garcea G and Maddern GJ (2009) Liver failure after major hepatic resection. J Hepatobiliary Pancreat Surg 16, 
145-155

3. Schmeding M, Boas-Knoop S, Lippert $S$ et al (2008) Erythropoietin promotes hepatic regeneration after extended liver resection in rats. J Gastroenterol Hepatol 23, 1125-1131

4. Greif F, Ben-Ari Z, Taya R et al (2010) Dual effect of erythropoietin on liver protection and regeneration after subtotal hepatectomy in rats. Liver Transpl 16, 631-638

5. Bockhorn M, Fingas CD, Rauen $U$ et al (2008) Erythropoietin treatment improves liver regeneration and survival in rat models of extended liver resection and living donor liver transplantation. Transplantation 86, 1578-1585

6. An S, Soe K, Akamatsu M, Hishikawa Y and Koji T (2012) Accelerated proliferation of hepatocytes in rats with iron overload after partial hepatectomy. Histochem Cell Biol $138,773-786$

7. Seehofer D, Neumann UP, Schirmeier A et al (2008) Synergistic effect of erythropoietin but not G-CSF in combination with curcumin on impaired liver regeneration in rats. Langenbecks Arch Surg 393, 325-332

8. Michalopoulos GK and DeFrances MC (1997) Liver regeneration. Science 276, 60-66

9. Tao Y, Wang M, Chen E and Tang H (2017) Liver regeneration: Analysis of the main relevant signaling molecules. Mediators Inflamm 2017, 4256352

10. Jelkmann W (2004) Molecular biology of erythropoietin. Intern Med 43,649-659.

11. Ghezzi P and Brines M (2004) Erythropoietin as an antiapoptotic, tissue-protective cytokine. Cell Death Differ 11, S37-44

12. Fan H, Villegas $\mathrm{C}$ and Wright JA (1996) A link between ferritin gene expression and ribonucleotide reductase R2 protein, as demonstrated by retroviral vector mediated stable expression of R2 cDNA. FEBS Lett 382, 145-148

13. Kuma S, Inaba M, Ogata $\mathrm{H}$ et al (1990) Effect of human recombinant interleukin- 6 on the proliferation of mouse hepatocytes in the primary culture. Immunobiology 180, 235-242

14. Kang LI, Mars WM, and Michalopoulos GK (2012) Signals and cells involved in regulating liver regeneration. Cells 1 , 1261-1292

15. Huh CG, Factor VM, Sanchez A, Uchida K, Conner EA and Thorgeirsson SS (2004) Hepatocyte growth factor/ c-met signaling pathway is required for efficient liver regeneration and repair. Proc Natl Acad Sci U S A 101, 4477-4482

16. Yokoyama $Y$, Nagino $M$ and Nimura $Y$ (2007) Mechanisms of hepatic regeneration following portal vein embolization and partial hepatectomy: a review. World J Surg 31, 367-374

17. Fisette A, Hassanain M, Metrakos P et al (2012) High-dose insulin therapy reduces postoperative liver dysfunction and complications in liver resection patients through reduced apoptosis and altered inflammation. J Clin Endocrinol Metab 97, 217-226

18. Pawlik TM, Poon RT, Abdalla EK et al (2005) Critical appraisal of the clinical and pathologic predictors of survival after resection of large hepatocellular carcinoma. Arch Surg 140, 450-457

19. Mollbrink A, Holmstrom P, Sjostrom M, Hultcrantz R, Eriksson LC and Stal P (2012) Iron-regulatory gene expression during liver regeneration. Scand J Gastroenterol 47, 591-600

20. Pachówka M, Zegarska J, Ciecierski $R$ and KorczakKowalska G (2008) The role of IL-6 during the late phase of liver regeneration. Ann Transplant 13, 15-19

21. Cui Y, König J, Leier I, Buchholz U and Keppler D (2001) Hepaticuptake of bilirubin and its conjugates by the human organic anion transporter SLC21A6. J Biol Chem 276, 9626-9630

22. Lehmann K, Tschuor C, Rickenbacher A et al (2012) Liver failure after extended hepatectomy in mice is mediated by a p21-dependent barrier to liver regeneration. Gastroenterology 143, 1609-1619

23. Koniaris LG, McKillop IA and Schwartz SI (2003) Liver regeneration. J Am Coll Surg 197, 634-659

24. Balleari E, Clavio M, Arboscello E et al (2011) Weekly standard doses of rh-EPO are highly effective for the treatment of anemic patients with low-intermediate 1 risk myelodysplastic syndromes. Leuk Res 35, 1472-1476

25. Ehrenreich H, Weissenborn K, Prange H et al (2009) Recombinant human erythropoietin in the treatment of acute ischemic stroke. Stroke 40, e647-656

26. Danielson J (2004) Structure, chemistry, and pharmacokinetics of intravenous iron agents. Am Soc Nephrol 15, S93-98

27. Wikström B, Bhandari S, Barany P et al (2011) Iron isomaltoside 1000: a new intravenous iron for treating iron deficiency in chronic kidney disease. J Nephrol 24, 589-596

28. Kalra PA and Bhandari S (2016) Efficacy and safety of iron isomaltoside (Monofer $(\mathbb{R})$ ) in the management of patients with iron deficiency anemia. Int J Nephrol Renovasc Dis 9, 53-64

29. Higgins GM and Anderson RM (1931) Experimental pathology of the liver. 1. Restoration of the liver of the white rat following partial surgical removal. Arch Pathol 12, 186-202

30. Meier M, Andersen KJ, Knudsen AR, Nyengaard JR, Hamilton-Dutoit S and Mortensen FV (2016) Liver regeneration is dependent on the extent of hepatectomy. J Surg Res 205, 76-84 